\title{
Shigesada et al. (1986) and population spread in heterogeneous environments
}

\author{
Frithjof Lutscher \\ Department of Mathematics and Statistics \\ Department of Biology \\ University of Ottawa \\ Ottawa, ON, K1N6N5, Canada
}

July 11, 2019

Studying the phenomenon of spatial spread in ecology and evolution has a long and distinguished history, starting with the work by Fisher (1937) on the advance of an advantageous gene and by Skellam (1951) on the spread of a population into previously unoccupied habitat. The overwhelming majority of models for these phenomena consider a homogeneous landscape in which individuals move randomly (modeled by diffusion) and interact locally (modeled by a differential equation). The resulting reaction-diffusion equations for the population density have spurred an impressive amount of research that ranges from mathematical analysis to model fitting, and have informed much of the modern theory in spatial ecology (e.g., Cantrell and Cosner (2013); Lewis et al. (2016)). Traveling waves are a special class of solutions of reaction-diffusion equations where a fixed density profile travels in a given direction at a constant speed. Traveling-wave analysis leads to the famous Fisher wave-speed formula

$$
c^{*}=2 \sqrt{D r},
$$

where $D$ is the diffusion coefficient and $r$ is the per capita growth rate at low density. Traveling waves and their speed are central to the modern study of biological invasions (Shigesada and Kawasaki, 1997; Lewis et al., 2016).

The groundbreaking contribution of Shigesada et al. (1986) was to formulate a reaction-diffusion model for spatial spread in a heterogeneous landscape that is complex enough to be biologically meaningful, yet simple enough to allow for explicit analysis of some of the most important quantities. Their 
landscape consists of two types of patches that alternate periodically in space. Movement behavior of individuals and population dynamics vary between but not within patches. For example, there are two diffusion coefficients, $D_{1}$ and $D_{2}$, and two low-density growth rates, $r_{1}$ and $r_{2}$, one for each patch. Instead of traveling waves, Shigesada et al. (1986) studied traveling periodic waves, spatial profiles that change periodically in space and time, but that still have a well-defined asymptotic speed. The authors considered source-sink landscapes, where one type of patch supports population growth $\left(r_{1}>0\right)$ and the other does not $\left(r_{2}<0\right)$. They derived an explicit formula for the minimal size of a source patch that ensures global population persistence. This result generalizes Skellam's result on the minimal size of a single good patch in an otherwise entirely inhospitable landscape. They also obtained an implicit formula for the speed of a traveling periodic wave and illustrated how it depends on the diffusion coefficients, the growth and death rates, and the lengths of the source and sink patches. For small patch sizes, they found an explicit wave-speed formula that generalizes (1), namely $c^{*}=2 \sqrt{\langle D\rangle_{h}\langle r\rangle_{a}}$ where

$$
\langle D\rangle_{h}=\frac{L_{1}+L_{2}}{\frac{L_{1}}{D_{1}}+\frac{L_{2}}{D_{2}}}, \quad \text { and } \quad\langle r\rangle_{a}=\frac{r_{1} L_{1}+r_{2} L_{2}}{L_{1}+L_{2}}
$$

denote the harmonic mean of the diffusion coefficients and the arithmetic mean of the growth rates, respectively.

Formulas like this equation for $c$ make this work applicable to many situations and give empirical researchers guidelines for the relevant spatial scales in their experiments. For example, Powell and Zimmermann (2004) used this modeling framework to describe localized caching of seeds by rodents and thereby offered one possible explanation for why certain tree species expanded their range so quickly after the last ice age (Reid's paradox). Powell and Zimmermann (2004) also gave an intuitive explanation in terms of residence times for why the harmonic mean appears in the equation.

Shigesada and co-workers generalized their central idea in a number of ways and applied it to various types of landscape heterogeneity in one- and two-dimensional space, e.g., Kinezaki et al. (2003). They inspired countless authors to contribute to the emerging theory of population spread in heterogeneous landscapes, e.g., by considering the competition of wild type and genetically modified organisms (Cruywagen et al., 1996), and river environments with pool-riffle structure (Lutscher et al., 2006). The mathematical approach by Shigesada et al. (1986) assumes that a traveling periodic wave exists and then derives its speed. Weinberger (2002) provided the first proof 
of the existence of a traveling wave in a slightly modified equation and thereby justified the calculations by Shigesada et al. (1986). More recently, the original authors expanded their approach again and included movement bias towards source patches (Kawasaki et al., 2012). Current experiments study the role of evolution on range expansion in such heterogeneous landscapes (Williams et al., 2016), and challenge the theory to catch up.

One curious observation in Shigesada et al. (1986) is that when sink patches are large, a population may be able to spread when individuals move slowly in sink patches, but may slow down and not spread when individuals move quickly in sink patches (their Figure 6c). This observation puzzled me when I first read their paper because it contradicted my intuition that faster individual movement should lead to higher population spread rates. It subsequently motivated a large portion of my own research since 2011. Together with G. Maciel, we realized how important individual movement behavior at an interface between two patch types is for the model outcome on the population level (Maciel and Lutscher, 2013). When individuals at an interface can choose the patch into which they move, the population density develops a jump at an interface, and the size of the jump depends on the movement rates in the adjacent patches as well as on the probability of moving into one or the other patch (Ovaskainen and Cornell, 2003). A careful analysis of the population spread rate with density jumps reveals that if the interface behavior of individuals follows some fairly general foraging strategies (e.g. movement in bad habitat should be faster and entry into bad habitat should be avoided), the "puzzling" outcome no longer arises. Moreover, the model with interface behavior and density jump gives a mechanistic explanation of the "puzzling" behavior in the model without interface behavior and without density jump that Shigesada et al. (1986) studied: If one wants to force the model with interface behavior to not have a density jump at the interface, one needs to assume that the probability of moving into a sink patch is low when the diffusion rate in a sink patch is high. Hence, when diffusion within sink patches is high, individuals rarely leave source patches, and the population spreads slowly or not at all (Maciel and Lutscher, 2013). Including behavioral details was not the goal of the original work of Shigesada et al. (1986) but has become since, e.g., in Shigesada et al. (2015), and shows once more how influential the original work continues to be. 


\section{References}

Cantrell, R.S., and Cosner, C. (2003). Spatial Ecology via Reation-Diffusion Equations. Wiley.

Cruywagen, G., Kareiva, P., Lewis, M., and Murray, J. (1996). Competition in a spatially heterogeneous environment: Modelling the risk of spread of a genetically engineered population. Theor. Popul. Biol., 49(1), 1-38.

Fisher, R. (1937). The advance of advantageous genes. Ann. Eugenics, 7, $355-369$.

Kawasaki, K., Asano, K., and Shigesada, N. (2012). Impact of directed movement on invasive spread in periodic patchy environments. Bull. Math. Biol., 74(6), 1448-1467.

Kinezaki, N., Kawasaki, K., Takasu, F., and Shigesada, N. (2003). Modeling biological invasions into periodically fragmented environments. Theor. Pop. Biol., 64, 291-302.

Lewis, M., Petrovskii, S., and Potts, J. (2016). The mathematics behind biological invasions. Springer.

Lutscher, F., Lewis, M., and McCauley, E. (2006). The effects of heterogeneity on population persistence and invasion in rivers. Bull. Math. Biol., 68(8), 2129-2160.

Maciel, G. and Lutscher, F. (2013). How individual response to habitat edges affects population persistence and spatial spread. Am. Nat., 182(1), $42-52$.

Ovaskainen, O. and Cornell, S.C. (2003). Biased Movement at a Boundary and Conditional Occupancy Times for Diffusion Processes. J. Appl. Prob., 40(3), 557-580.

Powell, J. and Zimmermann, N. (2004). Multiscale analysis of active seed dispersal contributed to resolving Reid's paradox. Ecology, 85(2), 490506.

Shigesada, N. and Kawasaki, K. (1997). Biological invasions: theory and practice. Oxford University Press.

Shigesada, N., Kawasaki, K., and Teramoto, E. (1986). Traveling periodic waves in heterogeneous environments. Theor. Popul. Biol., 30, 143-160. 
Shigesada, N., Kawasaki, K., and Weinberger, H. (2015). Spreading speeds of invasive species in a periodic patchy environment: effects of dispersal based on local information and gradient-based taxis. Japan J. Indust. Appl. Math., 32(3), 675-705.

Skellam, J. G. (1951). Random dispersal in theoretical populations. Biometrika, 38, 196-218.

Weinberger, H. F. (2002). On spreading speeds and traveling waves for growth and migration models in a periodic habitat. J. Math. Biol., 45, $511-548$.

Williams, J., Snyder, R., and Levine, J. (2016). The influence of evolution on population spread through patchy landscapes. Am. Nat., 188(1), 15-26. 